\title{
Can simple instructions to use spaced practice improve ability to remember a fact?: An experimental test using telephone numbers
}

\author{
THOMAS K. LANDAUER and BRIAN H. ROSS \\ Bell Laboratories, Murray Hill, New Jersey 07974
}

\begin{abstract}
As part of class demonstrations, college students were given single seven-digit telephone numbers and asked to memorize them according to one of two different sets of instructions. Control instructions asked the students to memorize the number as they "normally would." Experimental instructions recommended a form of spaced practice. After 2 weeks, recall was tested and confidence ratings obtained. The spaced-practice instructions produced significantly better recall than did control instructions. Confidence in incorrect answers was slightly lower in the experimental than in the control group, indicating that recommending the study method did not engender false confidence. Thus, merely prescribing spaced practice can help people memorize more effectively.
\end{abstract}

Laboratory research on human memory has made it clear that strategies for learning can vary widely in effectiveness. Thus, it seems plausible that simply telling people how to go about memorizing a new fact might be helpful. Optimal instructions would be ones that would lead to an improvement in a person's likelihood of correctly remembering a fact without leading to an unwarranted increase in confidence.

Our approach was aimed toward the identification of a technique that could be transmitted easily and quickly by written instructions. While there are a large number of factors that are known to affect learning, only some are applicable in this way. From this subset, we chose to employ spacing of practice for several reasons. First, variations in the distribution of practicewhether one rehearses in a single concentrated session or spreads practice over many shorter periods-are known to have consistent, sometimes quite dramatic effects on retention. In some situations well-spaced practice has been found to be as much as twice as effective as highly massed practice. The performances whose long-term retention is known to be improved by spacing of practice include motor skills, memorizing sets of related or unrelated letters, digits, or words, remembering associations between pairs of words or nonsense syllables, remembering the content of prose, and remembering pictures so as to be able to recognize them later. (See Hintzman, 1974, and Melton, 1970, for reviews of the experimental literature.) Second, it seems likely that people can learn how to use spaced practice without extensive training. This is in contrast to some of the

We thank C. S. Harris, D. E. Meyer, and S. Sternberg for helpful suggestions, and the many instructors and students of introductory psychology who provided the data. more elaborate mnemonic methods that are taught in memory courses and books. Third, there is reason to believe that spacing of practice might lead to more realistic self-appraisal of how well a new fact is known. For one thing, spaced practice can afford an opportunity to observe that one's memory is unreliable. For another, the effectiveness of massed practice is commonly overestimated. People often try to memorize a new fact by repeating it to themselves many times in succession immediately upon its receipt. They probably do this because it actually is effective in keeping a fact in mind over the short period during which it is rehearsed, or because they rightly believe that increased frequency of practice aids in retention, but are unaware that repetitions must be distributed to be effective. Thus, people who use massed rehearsal may not only remember less well than they might, but they may also tend to be overconfident. Indeed, Rothkopf (1963; Rothkopf \& Coke, 1966) found that even experienced educators, when judging the instructional effectiveness of text passages, tended to rate prose in which the repetition of a given piece of information was massed as better than those in which it was spaced, even though the actual measured effectiveness of the passages varied in the opposite way. It seemed not unlikely, then, that instructions to space practice might not only improve recall but also do so without unduly increasing false confidence.

Although the benefits of spaced practice have been amply demonstrated in laboratory experiments and distributed effort has often (e.g., Mace, 1968; Swain, 1917) been recommended as a study method, there does not appear to have been any direct research on the outcome of such advice. Thus, it was not obvious that the method could be applied successfully by prescription to a "real-life" task. Two important conditions that 
could easily be made more realistic, while still allowing adequately controlled study, were that the information need be remembered for days or weeks instead of minutes or hours, and that use of the method be induced by a simple set of instructions, rather than directly manipulated by the experimenter. The main motive for the experiment was, thus, to see whether the benefits of spacing would survive the conversions necessary to use it in a prescriptive manner for practical problems.

\section{METHOD}

The subjects were 708 students in introductory psychology classes at the State University of New York at Stony Brook, the University of Texas at Austin, and the College of William and Mary. At the end of a lecture hour each attending student was given a sheet of paper with instructions and a single telephone number to be memorized. The instructors of the classes told the students briefly that the sheets formed part of a demonstration to be carried out later in the term and asked for their cooperation. All sheets began with the same introductory paragraphs explaining and attempting to motivate the task. These paragraphs were as follows: "Telephone number memorizing experiment-Here is a small memorization exercise that will form the basis of a demonstration later in the term. The demonstration will be informative to you and should also yield data of actual scientific and practical value. Please read the following carefully and do as it asks. (Do not divulge the number on your sheet or discuss your instructions with anyone else in the class.) Imagine that you have just been given or looked up the new telephone number of a person whom you expect to call about twice a week for an indefinite period. Imagine also that, in addition to writing the number in an appropriate place, you wish to commit it to memory."

A random one-half of the sheets had a third paragraph instructing the reader to memorize the telephone number in the way he ordinarily would (control group), while the other one-half had a third paragraph instructing the reader to use a spaced practice method in which the number would be posted and looked at once or twice a day for a week (spaced-practice group). The experimental instructions were intended to be similar to ones that might be disseminated to telephone users. The two alternative forms of instruction were as follows: Experimental spaced-practice instructions-"On the basis of research results we recommend the following method of memorization. Write the number down (or keep this sheet). Post the number some place where you will see it once or twice a day for a week. This should be sufficient to enter the number permanently in your memory, and will be much more effective than concentrated rehearsal." Control instructions-"Try to memorize this number in exactly the same way that you normally would do so."

At the bottom of the page was a seven-digit number to be memorized, for example: "Here is the number 968-3125." For each group, there were eight different nonrepeating seven-digit numbers chosen such that no two numbers shared the same digit in the same place. The different numbers were randomly distributed among sheets of both kinds. Care was taken to avoid initial three digits that matched any of the exchange codes near the schools.

Two weeks after the sheets were handed out, recall data were collected by one of the authors (T.K. L.) who was giving a guest lecture. At the end of the lecture hour he passed out a sheet with a place for the student to record a recollection or best guess as to the telephone number and a brief questionnaire concerning the compliance of the student with the instructions, confidence in the correctness of the answer, and a calendar on which to estimate the time spent on memorizing each day for the preceding 2 weeks.

\section{RESULTS}

Since the main aim was to see whether a single prescriptive instruction to use spaced practice can improve the effectiveness of memorizing, the first question to be asked of the results is whether those receiving the specific instructions remembered their assigned number better than those who were instructed to memorize by their own method. Of the 362 subjects receiving the spaced-practice instructions, 263, or $72.7 \%$, correctly recalled the number. Of the 346 receiving control instructions, 211 , or $61.0 \%$, recalled the number correctly. This difference is statistically significant $\left(\chi^{2}=10.9, p<.005\right)$. Thus, it can be concluded that the spaced-practice instructions resulted in improved retention.

Let us now consider the false confidence question. We have said that a reasonable goal for memorization instructions is to increase correct recall without increasing erroneous guessing. While one wishes to remember a new acquaintance's name if possible, one may also be anxious not to call him or her by a wrong name. Similarly, one wants not only to remember telephone numbers correctly, but to avoid dialing wrong numbers.

First we note that the average confidence ratings were slightly, but not significantly, higher in the control group than in the experimental group for both correct and incorrect answers. For correct answers, mean confidence ratings were 4.77 and 4.69 (where 1 is "guess" and 5 is "very sure") for control and experimental groups, respectively. For incorrect answers, the corresponding means were 2.44 and 2.20 .

To further explore the probable effects of the experimental spaced-practice instructions, the data were analyzed as follows. Suppose people require a certain criterial level of confidence before hazarding an overt recall. Then those with lower confidence would give no answer, and those with higher confidence would give some correct and some erroneous responses, depending on the relation between confidence and recall accuracy. Accordingly, we give, in Table 1, the proportion of correct, incorrect, and nonresponses that would have been made assuming each confidence rating as the criterion for overt response. The table shows that, for any response criterion, the spaced-practice group would have made more correct responses, fewer incorrect responses, and fewer nonresponses than the control group.

It is also of interest to explore, insofar as we can, why the instructions worked. Did the students do as instructed? Did the resulting amount of spacing actually differ between control and experimental groups? If so, was it the spacing per se that helped, or something else?

The students indicated on the questionnaires the degree to which they followed the instructions, and were encouraged to make open-ended descriptions of their method of study. On a 5-point scale from "didn't follow the instructions at all" (scale value 1) to "followed the instructions very closely" (scale value 5), the 
Table 1

Percent of Students Who Would Have Taken Various Actions as a Function of Different Criteria for Attempted Responding

\begin{tabular}{|c|c|c|c|c|c|c|}
\hline \multirow[b]{2}{*}{ "Criterion for Responding" } & \multicolumn{3}{|c|}{ Experimental Instructions } & \multicolumn{3}{|c|}{ Control Group } \\
\hline & $\begin{array}{l}\text { Respond } \\
\text { Incorrectly }\end{array}$ & $\begin{array}{l}\text { Respond } \\
\text { Correctly }\end{array}$ & None & $\begin{array}{l}\text { Respond } \\
\text { Incorrectly }\end{array}$ & $\begin{array}{l}\text { Respond } \\
\text { Correctly }\end{array}$ & None \\
\hline (1) "guess" or higher & 26.3 & 73.7 & 0 & 37.9 & 62.1 & 0 \\
\hline (2) or higher & 16.0 & 72.8 & 11.2 & 25.9 & 62.1 & 12.1 \\
\hline (3) or higher & 9.0 & 71.4 & 19.6 & 16.5 & 61.5 & 22.1 \\
\hline (4) or higher & 6.7 & 69.7 & 23.5 & 11.8 & 59.4 & 28.8 \\
\hline (5) "very sure" & 3.1 & 57.1 & 39.8 & 5.0 & 51.2 & 43.8 \\
\hline
\end{tabular}

Note-Totals are not identical to those for overall data given in text because 11 out of 708 subjects failed to provide confidence ratings.

average rating for the spaced-practice group was 2.41 and for the control group was 3.69. Clearly, the instructions were not followed perfectly or equally by the two groups.

It is somewhat more difficult to interpret the answers to the open-ended question concerning methods of practice. However, $18.2 \%$ of the students in the control group and $11.6 \%$ in the spaced-practice instructions group reported having done all their studying in a single session. By contrast, only $18.2 \%$ of those in the control group, but $46.4 \%$ of those in the spaced-practice group, reported studying on 3 or more different days. Not surprisingly, those who reported studying on 3 or more days were more likely to recall correctly than those who reported studying only in one session $\left(\chi^{2}=10.6\right.$, $\mathrm{p}<.005$ and $\chi^{2}=4.0, \mathrm{p}<.05$ for control and spacedpractice groups, respectively). To further characterize the study methods of the two groups, we calculated summary statistics for the time/day calendars on the questionnaire. In each group, $91 \%$ of the students made such ratings. Those in the control group reported studying the number a median total of $181 \mathrm{sec}$ on a mean of 2.7 different days. Those in the spaced-practice group reported studying a median total of $344 \mathrm{sec}$ on a mean of 4.0 days. The experimental instructions seem to have increased both the number of different days on which subjects studied and the amount of time spent on each of those days. As shown in Table 2, there were small but statistically significant positive correlations between both total time and number of days of reported studying and probability correct for both control and spacedinstruction groups. Partial correlations between these

Táble 2

Product-Moment Correlations Between Two Aspects of Reported Study Time and Probability of Correct Recall

\begin{tabular}{lcc}
\hline \multicolumn{1}{c}{ Variable } & Control & Spaced-Practice \\
Group & Group \\
\hline Number of different days on & $.204 \dagger$ & $.273 \dagger$ \\
which number was studied & $(.098)^{*}$ & $(.212) \dagger$ \\
Total time spent studying & $.190 * *$ & $.180 * *$ \\
& $(.063)$ & $(-.037)$ \\
\hline
\end{tabular}

Note-Numbers in parentheses are partial correlations with the other variable held constant. ${ }^{*} p<.05 \quad{ }^{* *} p<.01 \quad t p<.001$ two measures of reported studying and probability correct, with the other measures held constant, are consistent with the interpretation that the number of different days of studying was more influential than total study time.

\section{DISCUSSION}

The results reported here offer reasonably convincing evidence that mere instruction to use spaced practice can lead to increased probability of recall after a few weeks without leading to an increase in incorrect guessing. It is not completely certain from the results of this experiment that the improved recall of the spaced-practice instruction group can be attributed to spacing of practice per se rather than to some other effect of the instructions. The subjects reported considerably less than perfect compliance with the instructions, and their estimates of study time varied in total as well as in distribution. However, correlations with probability correct were higher with reported number of days studied than with total time studied. Moreover, in a pilot study we compared instructions to memorize a telephone number by five one/day spaced recitations with instructions to memorize by 10 consecutive recitations on a single day. Of 94 students who reported actually using the spacedpractice method as instructed in the pilot study, $60.6 \%$ remembered correctly a week later, while, of 82 students who said they followed the massed-practice instruction, only $44.6 \%$ remembered correctly $\left(x^{2}=3.94, p<.05\right)$. There is, thus, some reason to believe that "spacing" was at least an important part of the present successful instruction set.

The most important implication of the results for present purposes is that such instructions can actually improve the effectiveness of memorization. How such instructions work is, of course, interesting, but does not need to be definitively established before their use can be recommended. However, some gaps of unknown seriousness still separate the conditions of this experiment from those of many potential practical applications. For example, motives for learning were not necessarily the same for the students in the experiment as they would be for other users, and the time period over which the information was remembered may or may not be entirely representative. A final answer as to whether spaced-practice instructions are of substantial help in a particular application would require a trial.

Nonetheless, the present results make it clear that the benefits of spacing are not confined to artificial laboratory conditions. They can be realized through brief written instruction. And they can improve learning even in circumstances in which people have had ample opportunity to develop good methods of memorization on their own. Thus, in summary, it seems to us that, when asked how to memorize, a psychologist or teacher would be well justified to advise the use of spaced practice. 


\section{REFERENCES}

Hintzman, D. L. Theoretical implications of the spacing effect. In R. L. Solso (Ed.), Theories in cognitive psychology: The Loyola symposium. Potomac, Md: Lawrence Erlbaum. 1974.

MACE, C. A. The psychology of study. Baltimore, Md: Penguin Books. 1968.

Melton. A. W. The situation with respect to the spacing of repetitions and memory. Journal of Verbal Learning and Verbal Behavior. 1970, 9. 596-606.
Roтнкорғ, E. Z. Some observations on predicting instructional effectiveness by simple inspection. Journal of Programmed Instruction, 1963, 2, 19-20.

RothKopf, E. Z., \& Coke, E. U. Variations in phrasing and repetition interval and the recall of sentence materials. Journal of Verbal Learning and Verbal Behavior, 1966, 5, 86-91.

Swain. G. F. How to study. New York: McGraw-Hill, 1917.

(Received for publication May 23, 1977.) 